\title{
Rectus abdominis muscle atrophy after thoracotomy
}

\author{
Jang Hoon Lee, Seok Soo Lee
}

Department of Thoracic and Cardiovascular Surgery, Yeungnam University Hospital, Yeungnam University College of Medicine, Daegu, Korea

Received: October 19, 2019

Revised: November 4, 2019

Accepted: November 15, 2019

Corresponding author:

Seok Soo Lee

Department of Thoracic and

Cardiovascular Surgery, Yeungnam

University Hospital, Yeungnam

University College of Medicine,

170 Hyeonchung-ro, Namgu, Daegu

42415 , Korea

Tel: +82-53-620-3880

Fax: +82-53-626-8660

E-mail: andrea0710@naver.com
Intercostal nerve injury is known to occur during thoracotomy; however, rectus abdominis muscle atrophy has rarely been reported. We describe a 52 -year-old man who underwent primary closure of esophageal perforation and lung decortication via left thoracotomy. He was discharged 40 days postoperatively without any complications. He noticed an abdominal bulge 2 months later, and computed tomography revealed left rectus abdominis muscle atrophy. We report thoracotomy induced denervation causing rectus abdominis muscle atrophy.

Keywords: Intercostal nerves; Muscle denervation; Muscular atrophy; Rectus abdominis; Thoracotomy

\section{Introduction}

Muscle atrophy observed after abdominal surgery is often attributable to direct muscle injury caused by incisions and sutures, as well as denervation of and reduced blood supply to the affected muscle $[1,2]$. However, abdominal muscle atrophy is uncommon after thoracotomy. We report a case of rectus abdominis muscle atrophy after left thoracotomy via the 9th intercostal space (ICS).

\section{Case}

All authors declare that written informed consent was obtained from the patient for publication of this case report and accompanying images.

A 52-year-old man was referred to our hospital with acute mediastinitis, empyema, and thoracotomy incision site infection. The patient underwent surgical treatment for esophageal rupture at another hospital, 9 days prior to presentation. On admission, the patient was in a state of septic shock; therefore, we performed

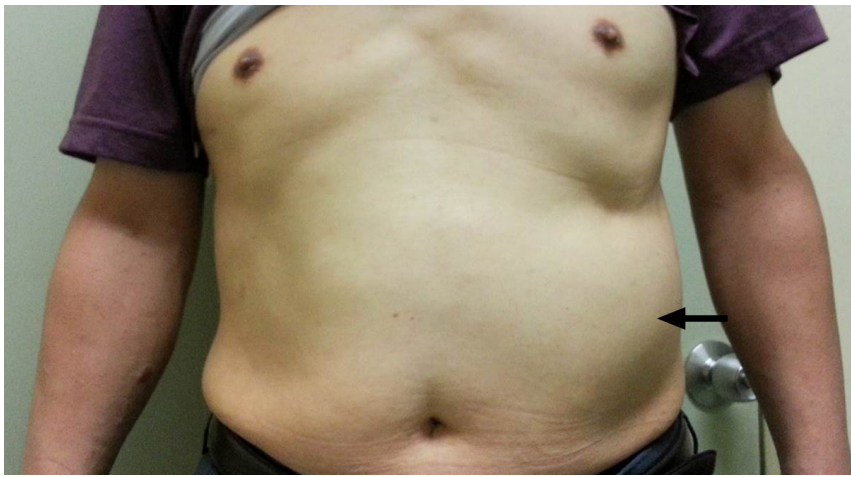

Fig. 1. Photograph of the left rectus abdominis muscle atrophy. The left lateral abdominal wall bulging is present (arrow).

emergency left thoracotomy via the previous incision site in the 9th ICS, and primary repair was performed. Three chest tubes were inserted into the 9th and 10th ICS for drainage. The patient's postoperative recovery was uneventful without esophageal leakage and/or wound complications. Owing to turbidity of the drained pleural fluid, we performed saline irrigation of the pleural

Copyright(C 2020 Yeungnam University College of Medicine

This is an Open Access article distributed under the terms of the Creative Commons Attribution Non-Commercial License (http://creativecommons.org/licenses/by-nc/4.0/) which permits unrestricted non-commercial use, distribution, and reproduction in any medium, provided the original work is properly cited. 

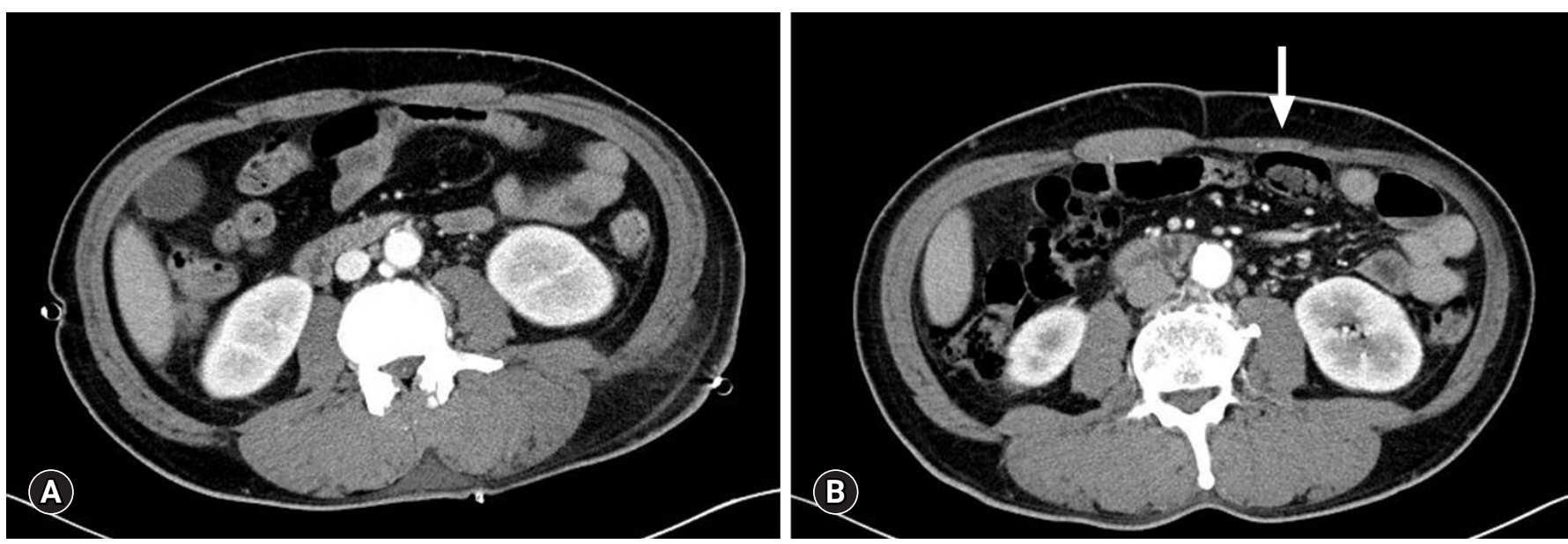

Fig. 2. (A) Preoperative abdominal computed tomography (CT) scan shows normal symmetric rectus abdominis. (B) Follow-up CT scan reveals a flattening of the left side rectus abdominis (arrow).

cavity through the chest tubes over several weeks. He was discharged in a good condition 6 weeks postoperatively. The patient returned 2 months later with a lump in the left anterior abdominal wall (Fig. 1), and we performed abdominal computed tomography (CT) at his follow-up visit. His preoperative CT revealed a normal symmetric rectus abdominis muscle (Fig. 2A); however, follow-up CT revealed flattening of the left rectus abdominis (Fig. 2B). CT confirmed left rectus abdominis muscle atrophy, which was presumably due to intercostal nerve injury during thoracotomy. The degree of intercostal nerve impairment is usually evaluated using electromyography, somatosensory evoked potentials, and assessment of tactile and pain thresholds to electrical stimulation [3]. However, in the present case, we diagnosed this condition based on the patient's clinical presentation and did not perform any other evaluation to assess nerve injury. Anatomically, lower thoracotomy is associated with a risk of rectus muscle paralysis because the rectus abdominis is innervated by the 7th through 12th intercostal nerves $[2,4]$. Thoracotomy may cause direct or indirect injury to intercostal nerves. Intercostal nerves that are not transected show axonal regeneration at a rate of $1-3 \mathrm{~mm} /$ day [5]. However, such nerve regeneration is usually asymmetrical and is associated with unaesthetic outcomes. Therefore, we expect that the nerve regeneration and recover the weakened rectus abdominis.

\section{Discussion}

The rectus abdominis muscle originates from the costal cartilages of the 5th, 6th, and 7th ribs and is inserted into the symphysis pubis and pubic crest. This muscle is innervated by anterior branches from the 7 th through 12 th intercostal nerves $[2,4]$. The incision made in the chest wall or rib spreading during thoracotomy may cause intercostal nerve injury [6], and such nerve injury can cause muscle paralysis [7]. In our case, left thoracotomy was performed via the 9th ICS and spreading two times; therefore, we conclude that rectus abdominis muscle atrophy was attributable to intercostal nerve injury.

\section{Acknowledgments}

\section{Conflicts of interest}

No potential conflict of interest relevant to this article was reported.

\section{ORCID}

Jang Hoon Lee, https://orcid.org/0000-0002-3990-888X

Seok Soo Lee, https://orcid.org/0000-0002-4402-0885

\section{References}

1. Vigneswaran Y, Poli E, Talamonti MS, Haggerty SP, Linn JG, Ujiki MB. Rectus abdominis atrophy after ventral abdominal incisions: midline versus chevron. Hernia 2017;21:619-22.

2. Matsumoto K, Noda T, Eguchi H, Iwagami Y, Akita H, Asaoka $\mathrm{T}$, et al. Atrophy of the rectus abdominis after left-side donor hepatectomy: comparison of upper abdominal midline vs mercedes incision. Transplant Proc 2019;51:1496-501.

3. Benedetti F, Vighetti S, Ricco C, Amanzio M, Bergamasco L, Casadio C, et al. Neurophysiologic assessment of nerve impairment in posterolateral and muscle-sparing thoracotomy. J Thorac Cardiovasc Surg 1998;115:841-7.

4. Antonescu I, Baird R. Paralysis of the rectus abdominis muscle after video-assisted thoracoscopic surgery for recurrent sponta- 
neous pneumothorax. J Pediatr Surg 2011;46:2397-400.

5. Beazley RM, Bagley DH, Ketcham AS. The effect of cryosurgery on peripheral nerves. J Surg Res 1974;16:231-4.

6. Rogers ML, Henderson L, Mahajan RP, Duffy JP. Preliminary findings in the neurophysiological assessment of intercostal nerve injury during thoracotomy. Eur J Cardiothorac Surg 2002;21:298-301.

7. Yamada M, Maruta K, Shiojiri Y, Takeuchi S, Matsuo Y, Takaba T. Atrophy of the abdominal wall muscles after extraperitoneal approach to the aorta. J Vasc Surg 2003;38:346-53. 